\title{
Blast Domination Number of Transformation Graphs of Linear and Circular Graphs
}

\begin{abstract}
A subset $S$ of $V$ of a non-trivial connected graph $G$ is called a Blast dominating set (BD-set), if $S$ is a connected dominating set and the induced sub graph $\langle V-S\rangle$ is triple connected. The minimum cardinality taken over all such Blast Dominating sets is called the Blast Domination Number (BDN) of $G$ and is denoted as, $\gamma_{c}^{t c}(G)$. In this article, let us mull over the generalized transformation graphs $G^{a b}$ and get hold of the analogous lexis of the Blast domination numbers for all the rage, transformation graphs, $G^{a b}$ and their complement graphs, $\overline{G^{a b}}$ for linear and circular graphs.
\end{abstract}

Keywords: Blast domination Number of a graph, Triple connected domination Number, Triple connected graph.

\section{INTRODUCTION}

Graphs considered at this juncture are simple, connected, undirected graphs. For customary graph theoretic notation, we refer to West [1] whilst for jargon allied to domination in graphs readers can refer to Haynes et al. [10]. The concept of triple connected graph was introduced by J.Paulraj Joseph in [8]. Triple connected domination number of a graph was introduced by G.Mahadevan et.al in [2]. Recently, massive number of varieties of various triple connected domination parameters were introduced by G.Mahadevan et.al.,

Provoked by all the above said triple connected domination parameters, in [3], freshly the conception of Blast domination number of a graph was introduced by G.Mahadevan, A.Ahila and SelvamAvadayappan with relevance $[3,4,5,6,7]$. In this paper, and fragment, we flog out on the subject of the performance of the Blast domination number in excess of the transformation graphs and their complement graphs of some standard graphs. The brainwave of this exertion is a piece of the applications of such transformation graphs.

\section{PRILIMINARY DEFINITIONS}

A subset $\mathrm{S}$ of $\mathrm{V}$ of a non-trivial connected graph $\mathrm{G}$ is called a Blast dominating set, if $\mathrm{S}$ is a connected dominating set and the induced sub graph $\langle V-S\rangle$ is triple connected. The minimum cardinality taken over all such Blast Dominating sets is called the Blast Domination Number of $G$ and is denoted as $\gamma_{c}^{t c}(G)$. Also, any Blast Dominating set with $\gamma_{c}^{t c}(G)$ vertices is called a $\gamma_{c}^{t c}$ - set of G.

Let $\mathrm{G}(\mathrm{V}, \mathrm{E})$ be any simple, non-trivial connected graph. Let us symbolize a graph of order $p$ and size $q$, in short as a $(p, q)$ graph. Let $\alpha, \beta$ be two elements of $V(G) \cup E(G)$.We say the associativity of $\alpha$ and $\beta$ is ' $f$ ' if they are either adjacent or incident in $\mathrm{G}$, to boot is ' - '. Let $a b$ be a 2-

Revised Manuscript Received on December 20, 2019.

* Correspondence Author

A.Ahila*, Mathematics, Kalasalingam Academy of Research and Education, Virudhunagar, India. Email: dr.aaasundar@gmail.com permutation of the set, $\{+,-\}$. Now it is defined as $\alpha$ and $\beta$ matches up to the first structure of $a b$ if both $\alpha$ and $\beta$ are in $V(G)$, whereas $\alpha$ and $\beta$ matches up to the second structure of $a b$ if both $\alpha$ and $\beta$ are in $E(G)$. The generalized transformation graph $G^{a b}$ is being defined on the vertex set of $V(G) \cup E(G)$, two vertices $\alpha$ and $\beta$ of $G^{a b}$ are adjacent if and only if their associability in $\mathrm{G}$ is consistent with respect to the consequent term $a b$. Apparently, we get four graphical transformation of graphs, with regard to the four dissimilar 2permutations of $\{+,-\}$, viz., $G^{++}, G^{+-}, G^{-+}$and $G^{--}$.

In other words, four graphical transformation of the generalized transformation graph $G^{a b}$ are the graphs whose vertex set is $V(G) \cup E(G)$ and $\alpha, \beta \in V\left(G^{a b}\right), \alpha$ and $\beta$ are adjacent in $G^{a b}$, if and only if either [*] or [**] holds.

[*] $-\alpha, \beta \in V(G), \alpha$ and $\beta$ are adjacent in $\mathrm{G}$, when $a=+$, not adjacent when $a=-$

[**] - $\alpha \in V(G)$ and $\beta \in E(G), \alpha, \beta$ are incident in $\mathrm{G}$, when $b=+$, not incident when $b=-$

Therefore, the four graphical transformation of the generalized transformation graph $G^{a b}$ are $G^{++}, G^{+-}, G^{-+}$and $G^{--}$. The four graphical transformations are therefore defined as follows:

$\alpha, \beta \in V\left(G^{a b}\right), \alpha$ and $\beta$ are adjacent in $G^{a b}$, if

1. $\boldsymbol{G}^{++}-[*] \alpha, \beta \in V(G), \alpha$ and $\beta$ are adjacent in $\mathrm{G}$ $[* *] \alpha \in V(G)$ and $\beta \in E(G), \alpha, \beta$ are incident in $\mathrm{G}$

2. $\boldsymbol{G}^{+-}-[*] \alpha, \beta \in V(G), \alpha$ and $\beta$ are adjacent in $\mathrm{G}$ $[* *] \alpha \in V(G)$ and $\beta \in E(G), \alpha, \beta$ are not incident in $\mathrm{G}$

3. $\boldsymbol{G}^{-+}[*] \alpha, \beta \in V(G), \alpha$ and $\beta$ are not adjacent in $\mathrm{G}$ $[* *] \alpha \in V(G)$ and $\beta \in E(G), \alpha, \beta$ are incident in $\mathrm{G}$

4. $\boldsymbol{G}^{--}-[*] \alpha, \beta \in V(G), \alpha$ and $\beta$ are not adjacent in $\mathrm{G}$ $[* *] \alpha \in V(G)$ and $\beta \in E(G), \alpha, \beta$ are not incident in $\mathrm{G}$

\section{CORE RESULTS}

Blast Domination Number over Transformation Graphs: In this episode, we attain the Blast domination numbers for both the generalized transformation graphs, $G^{a b}$ and their complement graphs, $\overline{G^{a b}}$ forlinear and circular graphs.

We embark on the following propositions, considered necessary for the proof of our chief results.

Proposition 3.1: Allow $\mathrm{G}$ as a $(p, q)$ graph. Then the degrees of point and edge vertices in $G^{a b}$ are

(i) $d_{G^{+}}\left(v_{i}\right)=2 d_{G}\left(v_{i}\right)$ and $d_{G^{++}}\left(e_{i}\right)=2$

(ii) $d_{G^{+-}}\left(v_{i}\right)=q$ and $d_{G^{+-}}\left(e_{i}\right)=p-2$

(iii) $d_{G^{-}}\left(v_{i}\right)=p-1$ and $d_{G^{-+}}\left(e_{i}\right)=2$

(iv) $d_{G^{-}}\left(v_{i}\right)=p+q-1-2 d_{G}\left(v_{i}\right)$

and $d_{G^{-}}\left(e_{i}\right)=q-2$

Proposition 3.2:Allow $\mathrm{G}$ as a $(p, q)$ graph. Then the order of $G^{a b}$ is $(p+q)$ and the size of $G^{++}, G^{+-}, G^{-+}$and $G^{--}$are $3 q, q(p-1), q+\frac{p(p-1)}{2}$ and 
$\frac{p(p-1)}{2}+q(p-3)$ respectively.

Stimulated by this definition, we prepared to corroborate the subsistence of the Blast domination number in these generalized transformation graphs, $G^{a b}$ and their complement graphs, $\overline{G^{a b}}$ for paths and cycles.

Let us contemplate over the generalized transformation graphs $G^{a b}$ and obtain the analogous lexis of the Blast domination numbers for all the rage, transformation graphs, $G^{a b}$ and their complement graphs, $\overline{G^{a b}}$.

Theorem 3.3:Let $G(V, E)$ be the linear graph, $P_{p}$. Then the Blast domination number for all transformation graphs and their complement graphs are as follows:

a) Blast domination number does not exist for $P_{p}^{++}$. But, $\gamma_{c}^{t c}\left(\overline{P_{p}^{++}}\right)=2$.

b) $\gamma_{c}^{t c}\left(P_{p}^{+-}\right)=3, \forall p \geq 4$. While, $\gamma_{c}^{t c}\left(\overline{P_{p}^{+-}}\right)=$ $2,, \forall p \geq 4$.

c) $\gamma_{c}^{t c}\left(P_{p}^{-+}\right)=2 p-4, \forall p \geq 4$. Whereas, $\gamma_{c}^{t c}\left(\overline{P_{p}^{-+}}\right)=2, \forall p \geq 4$.

d) $\gamma_{c}^{t c}\left[P_{p}^{--}\right]=2, \forall p \geq 4$. Where, $\gamma_{c}^{t c}\left(\overline{P_{p}^{--}}\right)=$ $\left\lceil\frac{p}{2}\right\rceil, \forall p \geq 4$

Proof: Let $G(V, E)$ be the linear graph, $P_{p}$. Relating to, $P_{p}^{a b}$ and its complement graph, $\overline{P_{p}^{a b}}$, the vertex set is $\left\{v_{1}, v_{2}, v_{3}, \ldots v_{p}, e_{12}, e_{23}, e_{34}, \ldots e_{(p-1) p}\right\}$ and so $\left|V\left[P_{p}^{a b}\right]\right|=$ $\left|V\left[P_{p}^{a b}\right]\right|=2 p-1$.

Claim (a): The graph obtained for $P_{p}^{++}$is a triangular snake graph, for which Blast domination number does not exist.

Now considering the complement of $P_{p}^{++}$, ie., $\overline{P_{p}^{++}}$, for any $p \geq 3$, we choose $v_{1}$ foremost, which dominates $v_{3}, \ldots v_{p}, e_{23}, e_{34}, \ldots . e_{(p-1) p}$ with the exception of $v_{2}$ and $e_{12}$. So to facilitate dominating these two vertices, it is as much as necessary to choose secondly, $v_{p}$, which is neighboring $v_{1}$ as well $v_{2}$ and $e_{12}$. Therefore, $S=\left\{v_{1}, v_{p}\right\}$, such that the induced sub graph $\langle V-S\rangle$ is triple connected. Hence, $\gamma_{c}^{t c}\left(\overline{P_{p}^{++}}\right)=2, \forall p \geq 3$.

Claim (b): In $P_{p}^{+-}$, for any $p \geq 4$, the set $\left\{v_{1}, e_{23}, v_{4}\right\}$ forms the blast dominating set. Therefore, $\gamma_{c}^{t c}\left(P_{p}{ }^{+-}\right)=3, \forall p \geq 4$. While allowing for the complement of $P_{p}{ }^{+-}$, ie., $\overline{P_{p}^{+-}}$, the vertex $v_{1}$ is adjacent to $v_{3}, v_{4}, \ldots v_{p}$ and $e_{12}$, except $v_{2}, e_{i j}, \forall 2 \leq i \leq(p-1) ; 3 \leq j \leq p$. But $e_{12}$ which is adjacent to $v_{1}$ and all those vertices which are non-adjacent to $v_{1}$. Therefore, the set $\left\{v_{1}, e_{12}\right\}$ will satisfy our $\mathrm{BDN}$ sharpness. Hence, $\gamma_{c}^{t c}\left(\overline{P_{p}^{+-}}\right)=2, \forall p \geq 4$.

Claim (c):Let us now consider the graph, $P_{p}^{-+}$. Here, the blast dominating set is to be $\left\{v_{1}, v_{2}, v_{3}, \ldots v_{p-2}, v_{p}, e_{12}, e_{23}, e_{34}, \ldots e_{(p-3)(p-2)}\right\}$, such that its complement set is $\left\{v_{p-1}, e_{(p-2)(p-1)}, e_{(p-1) p}\right\}$. Hence, $\gamma_{c}^{t c}\left(P_{p}{ }^{+}\right)=(p-2)+1+(p-3)=2 p-4, \forall p \geq 4$.

In view of the complement graph of $P_{p}{ }^{-+}$, ie., $\overline{P_{p}^{-+}}$, the set $\left\{e_{12}, e_{(p-1) p}\right\}$ holds out $\gamma_{c}^{t c}-\operatorname{set}$ norms. Thus, $\gamma_{c}^{t c}\left(\overline{P_{p}^{-+}}\right)=$ $2, \forall p \geq 4$.

Claim $(d)$ : Let us now consider the graph, $P_{p}^{--}$. In this graph, it is obvious that the set $\left\{v_{1}, v_{p}\right\}$ is $\gamma_{c}^{t c}-$ set, $\forall p \geq 4$. Thus, $\gamma_{c}^{t c}\left(P_{p}^{--}\right)=2, \forall p \geq 4$.
Considering the complement graph of $P_{p}^{--}$, ie., $\overline{P_{p}^{--}}$, all $v_{i}{ }^{\prime} s, 1 \leq i \leq p$ form a path $P_{p}$ and all $e_{j k}{ }^{\prime} s, 1 \leq j \leq$ $(p-1) ; 2 \leq k \leq p$ form a path $P_{p-1}$. Moreover, each $e_{j k}$, $1 \leq j \leq(p-1) ; 2 \leq k \leq p \quad$ is adjacent to the corresponding $v_{j}{ }^{\prime} s, v_{k}{ }^{\prime} s$ and $e_{m n}{ }^{\prime} s$ for which $m(n) \neq$ $j(k)$. In such a pattern,

Case (i): When $p=4$, the set $\left\{e_{12}, e_{34}\right\}$ is the BD-set and so our BDN is 2.

Case (ii): When $p=5$, the set $\left\{e_{12}, e_{34}, e_{45}\right\}$ is the BD-set and so our BDN is 3 .

Case (iii): When $p=6$, the set $\left\{e_{12}, e_{34}, e_{56}\right\}$ is the BD-set and so our BDN is 3 .

Case (iv): When $p=7$, the set $\left\{e_{12}, e_{34}, e_{56}, e_{67}\right\}$ is the BD-set and so our BDN is 4.

Case (v):When $p=8$, the set $\left\{e_{12}, e_{34}, e_{56}, e_{78}\right\}$ is the $\mathrm{BD}$-set and so our BDN is 4.

Proceeding like this, we observe that, $\gamma_{c}^{t c}\left(\overline{P_{p}^{--}}\right)=$ $\left\lceil\frac{p}{2}\right\rceil, \forall p \geq 4$.

Theorem 3.4:Let $G(V, E)$ be the cycle graph, $C_{p}$. Then the Blast domination number for all transformation graphs and their complement graphs are as follows:

a) $\gamma_{c}^{t c}\left(C_{p}{ }^{++}\right)=2 p-3, \forall p \geq 3 \quad$. Whereas, $\gamma_{c}^{t c}\left(\overline{C_{p}{ }^{++}}\right)=3, \forall p \geq 4$.

b) $\gamma_{c}^{t c}\left(C_{p}^{+-}\right)=5, \forall p \geq 4$. While, $\gamma_{c}^{t c}\left(\overline{C_{p}^{+-}}\right)=$ $\{2$, when $p=3,4$

$\{$, when $p \geq 5$

c) $\gamma_{c}^{t c}\left(C_{p}{ }^{-+}\right)=\left\{\begin{array}{c}5, \quad \text { if } p=4 \\ {\left[\frac{p}{2}\right], \text { for all } p \geq 5}\end{array}\right.$. Whereas, $\gamma_{c}^{t c}\left(\overline{C_{p}{ }^{-+}}\right)=2, \forall p \geq 3$.

d) $\gamma_{c}^{t c}\left(G^{--}\right)=\left\{\begin{array}{l}5, \text { for } p=4 \\ 3, \text { otherwise }\end{array}\right.$ Whereas, $\gamma_{c}^{t c}\left(\overline{C_{p}{ }^{-}}\right)=\left\lceil\frac{p}{2}\right\rceil$, with cardinality at least 4.

Proof: Let $G(V, E)$ be the circular graph, $C_{p}$. With respect to, $C_{p}{ }^{a b}$ and its complement graph, $\overline{C_{p}{ }^{a b}}$.

Let

$V\left[C_{p}{ }^{++}\right]=V\left[\overline{C_{p}^{++}}\right]=$

$\left\{v_{1}, v_{2}, v_{3}, \ldots v_{p}, e_{12}, e_{23}, e_{34}, \ldots . e_{(p-1) p}, e_{p 1}\right\}$

Claim (a): Consider $C_{p}{ }^{++}$

Case (i): When $p=2, C_{p}{ }^{++} \cong C_{3}$, for which Blast domination number does not exist.

Case (ii): When $p=3, V\left[C_{3}{ }^{++}\right]=\left\{v_{1}, v_{2}, v_{3}, e_{12}, e_{23}, e_{31}\right\}$.

In this (Figure 1), $v_{1}$ adjacent $v_{2}, v_{3}, e_{12}, e_{31}$ but not $e_{23}$. Therefore, in order to dominate $e_{23}$, it is essential to choose either $v_{2}$ or $v_{3}$, which is connected to $v_{1}$. Let us choose $v_{2}$. By this selection, $e_{12}$ is being left isolated and so we need to choose $e_{12}$ also as our BD-set member. Thus, $\gamma_{c}^{t c}-$ set of $C_{3}{ }^{++}$is $\left\{v_{1}, v_{2}, e_{12}\right\} . \therefore \gamma_{c}^{t c}\left[C_{3}{ }^{++}\right]=3=2(3)-3$.

Published By:

Blue Eyes Intelligence Engineering 


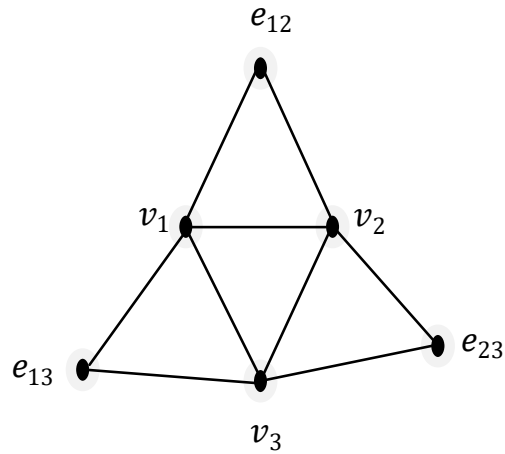

Fig. 1

Case (iii): When

$p=4$

$V\left[C_{4}{ }^{++}\right]=\left\{v_{1}, v_{2}, v_{3}, v_{4}, e_{12}, e_{23}, e_{34}, e_{41}\right\}$. In a similar way of argument, we get our BD-set as $\left\{v_{1}, v_{2}, v_{3}, e_{12}, e_{23}\right\} . \therefore$ $\gamma_{c}^{t c}\left[C_{4}^{++}\right]=5=2(4)-3,($ Fig. 2$)$

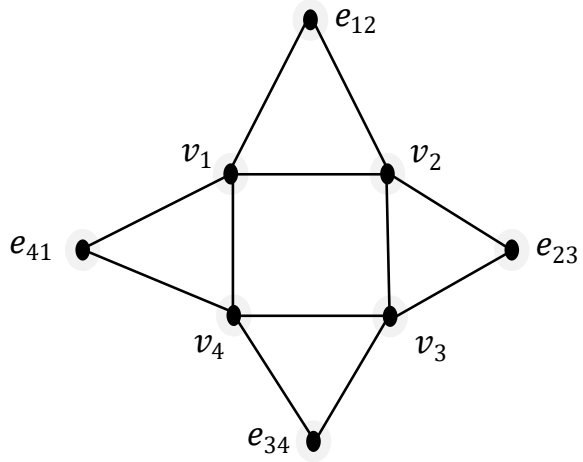

Fig. 2

Proceeding similar to this, with an analogous system of wiles, for any $p \geq 3, \gamma_{c}^{t c}-$ set of $C_{p}{ }^{++}$is $\left\{v_{1}, v_{2}, v_{3}, \ldots v_{p-1}, e_{12}, e_{23}, e_{34}, \ldots . e_{(p-2)(p-1)}\right\}=(p-1)+$ $(p-2)=2 p-3$.

In view of the complement of $C_{p}{ }^{++}$, ie., $\overline{C_{p}{ }^{++}}$, the induced sub-graph of all the edge vertices, $e_{i j}{ }^{\prime} s$ is $K_{p}$ and that of the vertices $v_{k}^{\prime} s$ is $\overline{C_{p}}$. Moreover, every $e_{i j}$ is adjacent to all $v_{k}^{\prime} s$ , such that $k \neq i$ or $j$. In such a construction, when $p=3$, the graph obtained is an uni-cyclic graph and so BDN does not exist. When $p \geq 4, e_{12}$ dominate all other $e_{i j}{ }^{\prime} s$ and all $v_{k}^{\prime} s$, such that $k \neq 1$ or 2 . Now, barely left non-dominated vertices are $v_{1}$ and $v_{2}$. But $e_{34}$ is adjacent to $e_{12}$ and dominate both $v_{1}$ and $v_{2}$. Therefore, it is better to choose $\left\{e_{12}, e_{34}\right\}$ as the minimal BD-set. Consequently, $\gamma_{c}^{t c}\left(\overline{C_{p}{ }^{-+}}\right)=2, \forall p \geq 3$.

Claim (b):Considering the graph, $C_{p}{ }^{+-}$, the set $\left\{v_{1}, v_{2}, v_{3}, e_{(p-2)(p-1)}, e_{(p-1) p}\right\}$ will be the minimum BD-set. Thus, $\gamma_{c}^{t c}\left(C_{p}{ }^{+-}\right)=5, \forall p \geq 4$.

Considering the complement graph of $C_{p}{ }^{+-}$, ie, $\overline{C_{p}{ }^{+-}}$, every $e_{12}$ dominate all $e_{i j}{ }^{\prime} s, v_{1}$ and $v_{2}$. While $v_{1}$ dominate all $v_{i}{ }^{\prime} s$, except $v_{2}$ and $v_{p}$. So in order to dominate, $v_{p}$, it is enough to choose $v_{3}$ adjacent to $v_{1}$. Thus for all $p \geq 5$, the set $\left\{v_{1}, v_{2}, e_{12}\right\}$ will be a minimum BD-set. But for $p=3$, we get $\left\{e_{12}, e_{31}\right\}$ and for $p=4$, we get $\left\{e_{12}, e_{34}\right\}$ as the minimal Blast dominating set. Hence, $\gamma_{c}^{t c}\left(\overline{C_{p}{ }^{+-}}\right)=$ $\{2$, when $p=3,4$

$\{$, when $p \geq 5$

Claim (c): Considering the graph, $\mathrm{C}_{p}{ }^{-+}$,

Case (i): When $p=3$, the graph obtained is a circular graph and so BDN does not exist.

Case (ii): When $p=4$, choosing $v_{1}$, that dominates $v_{3}, e_{12}, e_{41}$ but not $v_{2}, v_{4}, e_{23}, e_{34}$. So, next let us pick, $v_{3}$, which in turn dominate $e_{23}$ and $e_{34}$. Then to dominate $v_{2}$, it is obligatory to select $e_{23}$ and still $v_{4}$ remains non-dominated and hence we prefer choosing $v_{2}$. In such a state, the edge vertex $e_{12}$ is left isolated. Therefore, it is unavoidable to prefer $e_{12}$ also in the succession of our BD-set. Thus, the minimal Blast dominating set is $\left\{v_{1}, v_{3}, e_{23}, v_{2}, e_{12}\right\}$. Therefore, $\gamma_{c}^{t c}\left(C_{p}{ }^{+}\right)=5$, while $p=4$ Case (iii): When $p \geq 5$, it is crucial and clear with similar arguments, to consider the sequence of elements in our BD-set as $\left\{v_{1}, v_{3}, v_{5}, \ldots v_{p}\right\}$, when $p$ is odd and $\left\{v_{1}, v_{3}, v_{5}, \ldots v_{p-1}\right\}$, when $p$ is even. Then, $\gamma_{c}^{t c}\left(C_{p}{ }^{+}\right)=$ $\left\lceil\frac{p}{2}\right\rceil$, for all $p \geq 5$.

As a result, $\gamma_{c}^{t c}\left(C_{p}{ }^{-+}\right)=\left\{\begin{array}{c}5, \quad \text { if } p=4 \\ {\left[\frac{p}{2}\right\rceil, \text { for } \text { all } p \geq 5}\end{array}\right.$

Considering the complement of $\mathrm{C}_{p}{ }^{-+}$, the induced sub-graph obtained with vertices $\left\{e_{i j}, 1 \leq i, j \leq p\right\}$ is $K_{p}$ and each $e_{i j}$ adjacent rest other $e_{i j}{ }^{\prime} s$ including all $v_{k}^{\prime} s$, such that $k \neq i$ or $j$. And so to dominate $v_{i}$ and $v_{j}$, it is enough to select either $e_{(i-2)(j-2)}$ or $e_{(i+2)(j+2)}$, whichever exist. Thus, $\gamma_{c}^{t c}\left(\overline{C_{p}{ }^{-+}}\right)=2, \forall p \geq 3$

Claim (d): Consider, $C_{p}{ }^{--}, \forall p \geq 4$.

Case (i): When, $=4$, we attain the blast dominating set as $\left\{v_{1}, v_{3}, e_{12}, v_{4}, e_{23}\right\}$ constraint to the definition. Thus, $\gamma_{c}^{t c}\left[C_{p}{ }^{-}\right]=5$.

Case (ii): When $p \geq 5, v_{1}$ dominates all vertices except $v_{2}, v_{p}, e_{12}$ and $e_{p 1}$. So we choose $v_{3}$ adjacent to $v_{1}$, which in turn dominates $v_{p}, e_{12}$ and $e_{p 1}$. Next in order to dominate, $v_{2}$, it is enough to choose $v_{p}$, which is associated to $v_{3}$ and dominate $v_{2}$ as well. Thus, our $\gamma_{c}^{t c}-$ set of $C_{p}{ }^{--}$is for eternity, $\left\{v_{1}, v_{3}, v_{p}\right\} . \therefore \gamma_{c}^{t c}\left[C_{p}{ }^{--}\right]=3, \forall p \geq 5$.

Thus $\quad \gamma_{c}^{t c}\left[C_{4}{ }^{++}\right]=2 p-3, \forall p \geq 3 \quad, \quad$ whereas, $\gamma_{c}^{t c}\left[C_{p}{ }^{--}\right]=\left\{\begin{array}{l}5, \text { for } p=4 \\ 3, \text { otherwise }\end{array}\right.$.

Looking into the complement graph of ${C_{p}}^{--}$, ie., $\overline{C_{p}{ }^{--}}, \mathrm{S}$

While $p=4$, the set $\left\{e_{12}, e_{34}\right\}$ is the BD-set and so BDN is 2 . Whilst $p=5$, the set $\left\{e_{12}, e_{34}, e_{51}\right\}$ is the $\mathrm{BD}$-set and so $\mathrm{BDN}$ is 3. Once $p=6$, the set $\left\{e_{12}, e_{34}, e_{56}\right\}$ is the BD-set and so $\mathrm{BDN}$ is 3 . When $p=7$, the set $\left\{e_{12}, e_{34}, e_{56}, e_{71}\right\}$ is the $\mathrm{BD}$-set and so BDN is 4 . Thus by proceeding like this, we find that,

$\gamma_{c}^{t c}\left[\overline{C_{p}{ }^{-}},\right]=\left\lceil\frac{p}{2}\right\rceil, \forall p \geq 4$. 
Table- I: Consolidated values of BDN of Transformation graphs and their complements of Paths and Cycles

\begin{tabular}{|c|c|c|c|c|}
\hline \multirow{2}{*}{$\begin{array}{c}\text { G } \\
\backslash \\
G^{a b}\end{array}$} & \multicolumn{2}{|c|}{ Paths, $\boldsymbol{P}_{p}, \forall p \geq 4$} & \multicolumn{2}{|c|}{ Cycles, $C_{p}$} \\
\hline & $P_{p}^{a b}$ & $\begin{array}{l}\text { Compleme } \\
\text { nt of } \\
\qquad P_{p}^{a b}\end{array}$ & $C_{p}^{a b}$ & $\begin{array}{l}\text { Complement of } \\
\qquad \overline{C_{p}^{a b}}\end{array}$ \\
\hline$G^{++}$ & $\begin{array}{l}\text { Does not } \\
\text { exist }\end{array}$ & 2 & $2 p-3, \forall p \geq 3$ & $3, \forall p \geq 4$ \\
\hline$G^{+-}$ & 3 & 2 & $5, \forall p \geq 4$ & $\left\{\begin{array}{r}2, \text { when } p=3,4 \\
3, \text { when } p \geq 5\end{array}\right.$ \\
\hline $\boldsymbol{G}^{-+}$ & $2 p-4$ & 2 & $\left\{\begin{array}{c}5, \quad \text { if } p=4 \\
{\left[\frac{p}{2}\right], \text { for } \text { all } p \geq 5}\end{array}\right.$ & $2,, \forall p \geq 3$ \\
\hline $\boldsymbol{G}^{--}$ & 2 & $\left\lceil\frac{p}{2}\right\rceil$ & $\begin{array}{l}\left\{\begin{array}{l}5, \text { for } p=4 \\
3, \text { otherwise }\end{array}\right. \\
\forall p \geq 4\end{array}$ & $\left\lceil\frac{p}{2}\right\rceil, \forall p \geq 4$ \\
\hline
\end{tabular}

\section{ULTIMATE INTERPRETATION}

The domination and susceptibility of network are the two significant factors for the network system. We have introduced an important measure of vulnerability called Blast domination number. Here we have investigated the Blast domination number for splitting, m-splitting, co-splitting and degree spitting of paths, cycles, wheels and stars. The results reported here are sure to throw some glow in the track to work the same in larger graphs obtained from the given graph.

\section{REFERENCES}

1. D.B. West, Introduction To Graph Theory, 2/e, Prentice-Hall, New Delhi, India, 2003.

2. G.Mahadevan, A.Selvam, J.Paulraj Joseph and T.Subramanian, Triple connected domination number of a graph, International Journal of Mathematical Combinatorics, Vol.3 (2012), 93 - 104.

3. G.Mahadevan, A. Ahila and S. Avadayappan, Blast domination number of a graph, Middle East J. Sci. Res.,25(5) (2017), 977- 981.

4. G.G.Mahadevan, A..Ahila and S.Avaayappan, Blast Domination Number for $\vartheta$ - Obrazom, International Journal of Pure an Applied Mathematics, Volume 118, No.7, 2018, 111 - 117.

5. G.Mahadevan, A.Ahila, SelvamAvadayappan, Blast Domination Number of a graph Further Results, International Journal of Computational and Applied Mathematics, (2017) Volume 12, Number $1,307-312$.

6. G.Mahadevan, A.Ahila, SelvamAvadayappan, Blast Domination Number For Central and Total Graph of Star, Global Journal of Pure and Applied Mathematics, (2017) Volume 13, Number 2, 984 - 990.

7. G.Mahadevan, A.Ahila, SelvamAvadayappan, Blast Domination Number For Square and Cube Graphs of Graphs, in the Contemporary Studies in Discrete Mathematics - CSDM journal, Vol. 1, No. 1, 2017, pp. 31-35.

8. J.Paulraj Joseph, M.K.AngelJebitha, P.Chithra Devi and G.Sudhana, Triple connected graphs, Indian Journal of Mathematics and Mathematical Sciences, Vol.8, No.I(2012),61-75.

9. Lei $\mathrm{Yi}$, Baoyindureng $\mathrm{Wu}$, The Transformation Graph $\mathrm{G}^{++-}$, Australasian Journal of Combinatorics, Volume 44 (2009), Pages 37 42.

10. T. Haynes, S. Hedetniemi, and P. Slater, Fundamentals of Domination in Graphs, Marcel Dekker, New York, NY, USA, 1998.

11. V.R.Kulli, The Entire Edge Dominating Graph, in Advances in Domination Theory I, V.R.Kulli, ed., Vishwa International Pubications, Gulbarga, India, 155 (2012).

\section{AUTHORS PROFILE}

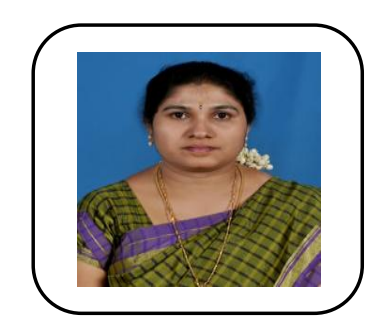

Ms. A.AHILA is working as a Professor of Mathematics in Kalasalingam Academy of Research and Education. She has published 9 publications in reputed journals. Her research field is new parameter Blast Dominatin Number of a Graph. She is a Life time member in ISTE and IAENG, BOM - KARE. She has 21 years of teaching experience, Reviewer - CSDM, Best Paper Award winner, Proud Rotarian. 